\title{
Dispersion Properties of Optical Polymers
}

\author{
N. Sultanova ${ }^{a}$, S. KASAROVA ${ }^{a, *}$ And I. NiKOLOV ${ }^{b}$ \\ ${ }^{a}$ Department of Physics, University "Assen Zlatarov", Bourgas, Bulgaria \\ ${ }^{b}$ Department of Optics and Spectroscopy, Faculty of Physics, University of Sofia, Bulgaria
}

\begin{abstract}
In this report dispersion properties of different types of optical polymers are discussed on the base of measured refractive indices and the Cauchy-Schott approximation. A number of dispersion curves are presented in the visible and near infrared spectral regions between 400 and $1060 \mathrm{~nm}$. A comparison with some optical glasses with similar refraction is performed. The nonlinear dependence of $\mathrm{d} n / \mathrm{d} \lambda$ of polymer materials and test glasses on the wavelength is calculated and analyzed. Normalized dispersion curves at $550 \mathrm{~nm}$ and $880 \mathrm{~nm}$ are presented to illustrate better the dispersion of the polymers in the considered spectral regions, separately. Abbe numbers are calculated to exhibit the mean and partial dispersion.
\end{abstract}

PACS numbers: 42.70.Jk, 78.20.Ci, 78.30.Jw

\section{Introduction}

Nowadays, optical polymers (OPs) are an excellent alternative to glasses not only in consumer but also in high quality optics $[1,2]$. Although the variety of optical glass materials exceeds those that are currently available for plastic optics, plastics are preferred materials when integrating multiple features and elements, low weight and high impact resistance are needed.

Chemical companies produce various types of OPs but some of them have close refractive and dispersive data [3]. Many of the offered plastics are available in increasing number of grades that affect such important characteristics as improved thermal stability or lower moisture absorption which relate directly to their index of refraction. The practical use of a given material in optical design requires precise data of its refractive index and dispersion behaviour. Optical glass catalogues provide refractive index data at a wide range of frequently used wavelengths and Abbe numbers at $d$ - and $e$-spectral lines as a measure of dispersion [4]. However, the information on refractive and dispersion properties of optical polymers is yet scanty.

We have measured different types of plastic materials in the visible (VIS) and near infrared (NIR) regions assuring accuracy better than $\pm 10^{-3}[3,5]$. The obtained refractive values were compared with the data from optical software libraries and glass catalogues. In this report a detailed comparison of the dispersion properties of the examined polymers is presented. Abbe numbers of mean and partial dispersion and dispersion charts are calculated. Some additional criteria for better comparison between the polymers are introduced.

\section{Measurement of optical polymers}

In this study we apply the deviation angle method for refractive index measurement described in detail in

\footnotetext{
* corresponding author; e-mail: kasarova_st@yahoo.com
}

our previous papers $[3,5]$. We have measured the principal optical plastics and some development materials produced by chemical companies as Eastman Chemical Company (ECC), Bayer, Nippon Zeon Company, etc. All measurement samples were prepared in the form of plates with thickness from $2.5 \mathrm{~mm}$ to $5.5 \mathrm{~mm}$. The indices of refraction were measured with the aid of the V-type SF3 glass prism (VoF3 prism) on the Carl Zeiss Jena Pulfrich-Refractometer (PR2) which works with three spectral lamps for the VIS region. Additional goniometric setup with the same prism, white lighting module including interference filters (IFs) and a photodetector device was used. The transmission maxima of the applied IFs are as follows - 548, 589, 659, $703 \mathrm{~nm}$ for the VIS region, and $752,804,833,879$ and $1052 \mathrm{~nm}$ for the NIR region. A number of OP specimens have been measured using a $\mathrm{He}-\mathrm{Ne}$ laser at $632.8 \mathrm{~nm}[6]$. We used silicon oil with a refractive index $n_{\mathrm{D}}=1.5601$ as a contacting immersion during the $\mathrm{OPs}$ measurements. The $\mathrm{K}-\mathrm{Hg}-\mathrm{J}$ solution with an index $n_{\mathrm{e}}=1.7301$ was applied in cases of high refractive samples. The measuring temperature was maintained at $20^{\circ} \mathrm{C}$ by a thermostat and temperature regulation was possible with stability of $0.2{ }^{\circ} \mathrm{C}$. The metrological tests pointed out that our measurements of OPs indices guarantee the accuracy of \pm 0.001 . Nonlinear dependence of the differences between the refractometric and goniometric measured data on the wavelength was noticed [3]. Highest values of deviations occur in VIS and lowest in NIR region.

The obtained values of refractive indices of the examined OPs at measuring wavelengths are presented in Table. In the last two columns Abbe numbers $\nu_{\mathrm{d}}$ and $\nu_{879} \mathrm{~nm}$ are given to characterize the dispersion properties in the VIS and NIR ranges, respectively. Mean and partial dispersion in the NIR region are calculated by the following expressions:

$$
\nu_{\mathrm{d}}=\frac{n_{\mathrm{d}}-1}{n_{\mathrm{F}}-n_{\mathrm{C}}} \quad \text { and } \quad \nu_{879}=\frac{n_{879}-1}{n_{703}-n_{1052}} .
$$

Here $n_{\mathrm{d}}, n_{\mathrm{F}}$ and $n_{\mathrm{C}}$ are measured indices at 587.56, 486.13 and $656.27 \mathrm{~nm}$ in VIS and $n_{703}, n_{879}, n_{1052}-$ 
measured values in NIR region. Grouping of OPs can be accomplished in accordance with their refractive index values and dispersion characteristics. Low refractive materials as acrylics, cellulose, low styrene, Optorez and Zeonex have higher Abbe numbers, while OPs with refractive indices above 1.55 have magnitude of $\nu_{\mathrm{d}}$ less than 40

Measured refractive indices and Abbe numbers of optical polymers.

TABLE

\begin{tabular}{l|c|c|c|c|c|c|c|c|c|c}
\hline \multirow{2}{*}{$\begin{array}{l}\text { Optical } \\
\text { polymers }\end{array}$} & \multicolumn{7}{|c|}{ Wavelength [nm] } & \multicolumn{3}{c}{$\begin{array}{c}\text { Abbe } \\
\text { numbers }\end{array}$} \\
\cline { 2 - 11 } & 436.8 & 486.1 & 587.6 & 632.8 & 703 & 833 & 879 & 1052 & $\nu_{\mathrm{d}}$ & $\nu_{879}$ \\
\hline PMMA & 1.502 & 1.497 & 1.491 & 1.489 & 1.486 & 1.484 & 1.483 & 1.481 & 59.2 & 96.7 \\
PC & 1.612 & 1.599 & 1.585 & 1.580 & 1.575 & 1.569 & 1.568 & 1.565 & 29.1 & 54.6 \\
PS & 1.617 & 1.606 & 1.592 & 1.587 & 1.582 & 1.577 & 1.576 & 1.572 & 30.5 & 56.4 \\
SAN & 1.588 & 1.578 & 1.567 & 1.563 & 1.558 & 1.554 & 1.553 & 1.550 & 35.4 & 66.6 \\
NAS-21 & 1.593 & 1.584 & 1.571 & 1.568 & 1.564 & 1.558 & 1.557 & 1.554 & 35.5 & 56.3 \\
CTE Rich. & 1.602 & 1.593 & 1.580 & 1.576 & 1.571 & 1.566 & 1.565 & 1.562 & 32.8 & 58.3 \\
S-low styr. & 1.531 & 1.525 & 1.516 & 1.514 & 1.512 & 1.509 & 1.508 & 1.506 & 44.9 & 79.4 \\
Optorez 1330 & 1.522 & 1.516 & 1.509 & 1.508 & 1.505 & 1.503 & 1.502 & 1.498 & 52.0 & 71.7 \\
Zeonex E48R & 1.543 & 1.538 & 1.531 & 1.528 & 1.526 & 1.523 & 1.522 & 1.520 & 56.5 & 100.5 \\
Bayer & 1.612 & 1.600 & 1.586 & 1.581 & 1.576 & 1.571 & 1.570 & 1.566 & 30.0 & 54.3 \\
Acrylic & 1.502 & 1.498 & 1.492 & 1.490 & 1.488 & 1.485 & 1.485 & 1.483 & 57.8 & 96.9 \\
Polyacrylate & 1.506 & 1.499 & 1.494 & 1.492 & 1.491 & 1.489 & 1.488 & 1.486 & 63.3 & 97.6 \\
Styrene & 1.534 & 1.527 & 1.519 & 1.516 & 1.513 & 1.510 & 1.509 & 1.507 & 42.9 & 77.1 \\
Cellulose & 1.480 & 1.477 & 1.471 & 1.469 & 1.466 & 1.463 & 1.463 & 1.461 & 54.1 & 84.1 \\
EBM-copol. & 1.586 & 1.575 & 1.561 & 1.558 & 1.554 & 1.548 & 1.547 & 1.545 & 31.4 & 58.8
\end{tabular}

\section{Discussion on dispersion properties of OPs}

On the base of the measured results some values of the optical material's refractive index can be calculated using the well known dispersion equations [3]. We have chosen the Cauchy-Schott relation which is valid for transparent media in their region of normal dispersion $[3,5]$ :

$$
n_{\lambda}^{2}=A_{1}+A_{2} \lambda^{2}+\frac{A_{3}}{\lambda^{2}}+\frac{A_{4}}{\lambda^{4}}+\ldots
$$

We have compared this approximation with the well-known Sellmeier relation. Our calculations have shown that usage of six dispersion coefficients in the CauchySchott approximation assures calculation accuracy better than $10^{-4}[5]$. We have realized a program OptiColor that computes the dispersion coefficients with the aid of a system consisting of six linear equations. On the base of the obtained coefficients dispersion curves are calculated and drawn with the aid of our OptiColor program. The charts of the refractive index of some OPs versus the wavelength between 435 and $1060 \mathrm{~nm}$ are illustrated in Fig. 1. The presented plots are smooth and monotonously decreasing in the range of normal dispersion which is in unison with the approximation, given in Eq. (2).

Wavelength dependence of $\mathrm{d} n / \mathrm{d} \lambda$ assures more detailed examination of the dispersion behaviour of the studied materials in various parts of the spectrum. The plots of $|\mathrm{d} n / \mathrm{d} \lambda|$ versus $\lambda$ of OPs and some Schott

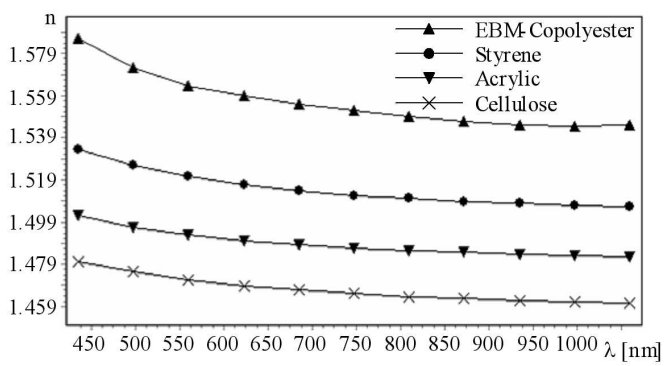

Fig. 1. Dispersion curves of ECC polymers.

optical glasses are shown in Fig. 2. The graphs decrease monotonously except for the CTE-Richardson curve which measured index at the blue line is probably influenced by birefringence and internal stresses in the sample. Bayer polymer as a polycarbonate type material shows the highest dispersion (Fig. 2a). CTE-Richardson plastic has lower but similar dispersion and both curves overlap completely at the end of VIS and NIR regions. Cellulose has the most fluently falling with the wavelength dispersion curve (Fig. 2b). As it can be seen, Acrylic and N-BK7 have close dispersion and the graphs of Optorez 1330 and N-BAK4 almost coincide in the considered spectral region.

A better comparison and vizualization of the dispersion properties of the examined materials is achieved by 


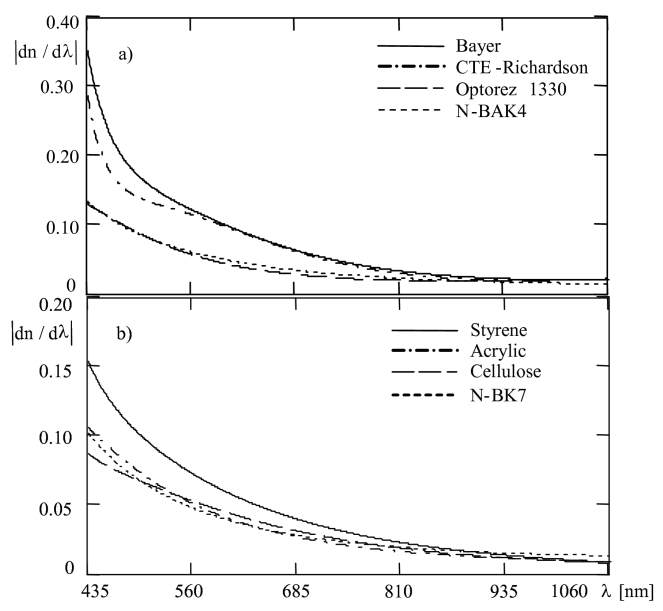

Fig. 2. Wavelength dependence of $|\mathrm{d} n / \mathrm{d} \lambda|$ of polymers compared with Schott glasses: (a) OPs trade-marks and N-BAK4; (b) ECC materials and N-BK7.

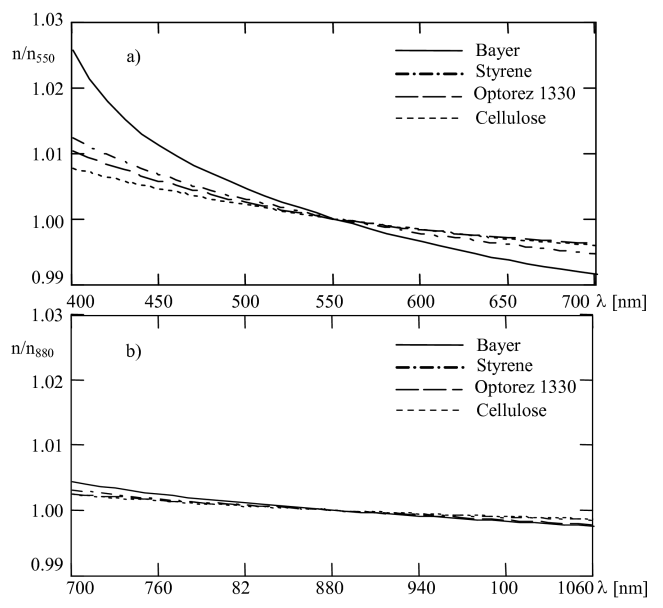

Fig. 3. Normalized dispersion curves of OPs at: (a) $550 \mathrm{~nm}$; (b) $880 \mathrm{~nm}$.

presentation of the normalized dispersion curves to the refractive index value at definite wavelength, e.g. $550 \mathrm{~nm}$ [7] for VIS and $880 \mathrm{~nm}$ for NIR, respectively. As shown in Fig. 3 even slight differences in the curves' behaviour are well perceivable and easy distinguishable. More considerable differences in dispersion properties are observed in the VIS area - Fig. 3a. Bayer polymer reveals the highest dispersion in the whole examined range, while the normalized curves of the low dispersive Optorez 1330 and Cellulose coincide fairly beyond $550 \mathrm{~nm}$.

Normalized dispersion curves of the same materials in the NIR region are given in Fig. 3b. Though the scale of the graph is maintained, the dispersion properties of the polymers are quite close. OPs as styrene, Optorez 1330 and cellulose exhibit extremely flat wavelength dispersion making them well-situated for broad wavelength applications.

The presented results and computed charts make possible the comparison of the dispersion properties of the examined materials. The correctly computed dispersion charts should be equal-distant and monotonously decreasing in the range of normal dispersion (Fig. 1). Calculated and measured refractive data generate coinciding curves. High refractive OPs as PC, Bayer (Figs. 2a, 3a) demonstrate higher dispersion. Greatest variation of dispersion properties are noticed in the VIS region especially at lower wavelengths (Figs. 2 and 3a). Dispersion in the NIR range is not a limiting factor for all of the examined polymers where their normalized curves are extremely flat (Fig. 3b).

\section{References}

[1] J. Menendez, F. Erismann, M. Gauvin, Opt. Photon. News 10, 28 (1999).

[2] P. Tolley, Photon. Spectra, 76 (October 2003).

[3] N. Sultanova, I. Nikolov, C. Ivanov, Opt. Quant. Electron. 35, 21 (2003).

[4] http://www.us.schott.com/ .

[5] S. Kasarova, N. Sultanova, C. Ivanov, I. Nikolov, Opt. Mater. 29, 1481 (2007).

[6] I. Nikolov, N. Sultanova, S. Kasarova, Proc. SPIE 5830, 511 (2005).

[7] http://www.zeonex.com/ applications_zeonorfilm.asp/ . 